Dept. of Math. University of Oslo

$\begin{array}{lr}\text { Pure Mathematics } & \text { No. } 11 \\ \text { ISSN 0806-2439 } & \text { MarCH } 2005\end{array}$

\title{
The value of information in stochastic control and finance
}

\author{
Bernt Øksendal \\ Center of Mathematics for Applications (CMA) \\ Dept. of Mathematics, University of Oslo \\ Box 1053 Blindern, N-0316 Oslo, Norway \\ email: oksendal@math.uio.no \\ and \\ Norwegian School of Economics and Business Administration, \\ Helleveien 30, N-5045 Bergen, Norway
}

Revised May 2005

\begin{abstract}
We present an optimal portfolio problem with logarithmic utility in the following 3 cases:

(i) The classical case, with complete information from the market available to the agent at all times. Mathematically this means that the portfolio process is adapted to the filtration $\mathcal{F}_{t}$ of the underlying Brownian motion (or, more generally, the underlying Lévy process).

(ii) The partial observation case, in which the trader has to base her portfolio choices on less information than $\mathcal{F}_{t}$. Mathematically this means that the portfolio process must be adapted to a filtration $\mathcal{E}_{t} \subseteq \mathcal{F}_{t}$ for all $t$. For example, this is the case if the trader can only observe the asset prices and not the underlying Lévy process.

(iii) The insider case, in which the trader has some inside information about the future of the market. This information could for example be the price of one of the assets at some future time. Mathematically this means that the portfolio process is allowed to be adapted to a filtration $\mathcal{G}_{t} \supseteq \mathcal{F}_{t}$ for all $t$. In this case the associated stochastic integrals become anticipating, and it is necessary to explain what mathematical model it is appropriate to use and to clarify the corresponding anticipating stochastic calculus.
\end{abstract}

We solve the problem in all these 3 cases and we compute the corresponding maximal expected logarithmic utility of the terminal wealth. Let us call these quantities $V_{\mathcal{F}}, V_{\mathcal{E}}$ and $V_{\mathcal{G}}$, respectively. Then $V_{\mathcal{F}}-V_{\mathcal{E}}$ represents the loss of value due the loss of information in (ii), and $V_{\mathcal{G}}-V_{\mathcal{F}}$ is the value gained due to the inside information in (iii). 


\section{Introduction}

The Brownian motion process $B(t)=B(t, \omega) ; t \geq 0$ is a classical tool in mathematical finance. For example, the Black-Scholes model assumes that the price $S(t)$ of the risky asset is a geometric Brownian motion described by a stochastic differential equation of the form

$$
d S(t)=S(t)[\mu d t+\sigma d B(t)] ; \quad S(0)>0
$$

where $\mu$ and $\sigma \neq 0$ are constants.

In spite of the success of this model, it is clear that it does not fit the data well. To improve the model it has been suggested to use the more general Lévy process as an underlying, driving process rather than just $B(t)$. See e.g. Barndorff-Nielsen (1998), Eberlein (2001), Schoutens (2003). By definition a Lévy process on a filtered probability space $\left(\Omega, \mathcal{F},\left\{\mathcal{F}_{t}\right\}_{t \geq 0}, P\right)$ is a process $\eta(t)=\eta(t, \omega):[0, \infty) \times \Omega \rightarrow \mathbb{R}$ such that

(i) $\eta(\cdot)$ has stationary, independent increments

(ii) $\eta(0)=0$

(iii) $\eta$ is cadlag, i.e. the paths $t \rightarrow \eta(t)$ are right continuous with left limits.

We also assume

$$
E\left[\eta^{2}(t)\right]<\infty \text { for all } t \geq 0,
$$

where $E[\cdot]$ denotes the expectation with resect to the probability law $P$ of $\eta(\cdot)$. By the Lévy-Itô decomposition theorem (see Applebaum (2004)) any such Lévy process has the form

$$
\eta(t)=a t+c B(t)+\int_{\mathbb{R}} z \tilde{N}(d t, d z)
$$

where $a, c \in \mathbb{R}$ are constants, $B(t)$ is an $\mathcal{F}_{t}$-Brownian motion and

$$
\tilde{N}(d t, d z)=N(d t, d z)-\nu(d z) d t
$$

is the compensated Poisson random measure of $\eta$. Here $N([0, t], F)$ is the random measure giving the number of jumps of $\eta(\cdot)$ in the time interval $[0, t]$ and of size $\Delta \eta \in F$, where $F$ is a Borel set in $\mathbb{R}$ with $0 \notin \bar{F}$. The measure

$$
\nu(F)=E[N([0,1], F)]
$$

is called the Lévy measure of $\eta(\cdot)$.

If we replace $d B(\cdot)$ by $d \eta(\cdot)$ in (1.1) we get the equation

$$
d S(t)=S\left(t^{-}\right)\left[\mu d t+\sigma a d t+\sigma c d B(t)+\sigma \int_{\mathbb{R}} z \tilde{N}(d t, d z)\right] .
$$

It is now natural to go one step further and consider more general stochastic differential equations with unrelated coefficients of $d t, d B(t)$ and $\tilde{N}(d t, d z)$. Thus we arrive at the following general Lévy market model:

Consider a mathematical market consisting of the following two investment possibilities: 
(a) A risk free investment (e.g. a bond), whose unit price $S_{0}(t)$ at time $t$ is goverend by an equation of the form

$$
d S_{0}(t)=\rho(t) S_{0}(t) d t ; \quad S_{0}(0)=1 ; \quad 0 \leq t \leq T \text { (constant) }
$$

(b) A risky investment (e.g. a stock), whose unit price $S(t)$ at time $t$ is described by a stochastic differential equation of the form

$$
\begin{aligned}
& d S(t)=S\left(t^{-}\right)\left[\mu(t) d t+\sigma(t) d B(t)+\int_{\mathbb{R}} \theta(t, z) \tilde{N}(d t, d z)\right] \\
& S(0)>0 ; \quad 0 \leq t \leq T
\end{aligned}
$$

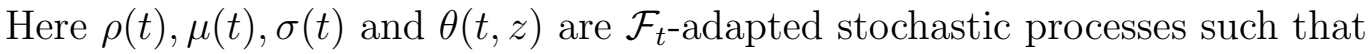

$$
E\left[\int_{0}^{T}\left\{|\rho(s)|+|\mu(s)|+\sigma^{2}(s)+\int_{\mathbb{R}} \theta^{2}(s, z) \nu(d z)\right\} d s\right]<\infty
$$

and

$$
\theta(t, z)>-1 \quad \text { for a.a. } t, z \text { with respect to } d t \times \nu(d z) \text {. }
$$

(This prevents $S(t)$ from jumping to 0 or a negative value.)

Then by the Itô formula for semimartingales the solution of (1.8) is

$$
\begin{aligned}
S(t) & =S(0) \exp \left[\int_{0}^{t}\left\{\mu(s)-\frac{1}{2} \sigma^{2}(s)-\int_{\mathbb{R}}(\theta(s, z)-\ln (1+\theta(s, z))) \nu(d z)\right\} d s\right. \\
& \left.+\int_{0}^{t} \sigma(s) d B(s)+\int_{0}^{t} \int_{\mathbb{R}} \ln (1+\theta(s, z)) \tilde{N}(d s, d z)\right] ; \quad 0 \leq t \leq T .
\end{aligned}
$$

We refer to Applebaum (2004), Jacod et al (2003), Øksendal et al (2004b) and Protter (2004) for more information about stochastic calculus for Lévy processes.

Suppose that a trader in this market is free to choose at any time $t$ the fraction $\pi(t)$ of her current wealth $X(t)$ to be invested in the risky asset. The corresponding wealth process $X(t)=X^{(\pi)}(t)$ will then satisfy the equation

$$
d X(t)=(1-\pi(t)) X(t) \rho(t) d t+\pi(t) X\left(t^{-}\right)\left[\mu(t) d t+\sigma(t) d B(t)+\int_{\mathbb{R}} \theta(t, z) \tilde{N}(d t, d z)\right]
$$

or

$$
\begin{aligned}
d X(t) & =X\left(t^{-}\right)[\{\rho(t)+(\mu(t)-\rho(t)) \pi(t)\} d t+\sigma(t) \pi(t) d B(t) \\
+ & \left.\pi(t) \int_{\mathbb{R}} \theta(t, z) \tilde{N}(d t, d z)\right] ; \quad 0 \leq t \leq T .
\end{aligned}
$$


For simplicity we put

$$
X(0)=1
$$

Suppose the information available to the trader at time $t$ is represented by a filtration (i.e. an increasing right continuous family of $\sigma$-algebras) $\mathcal{H}_{t} ; 0 \leq t \leq T$. This means that the portfolio process $\pi(t)$ is adapted to $\mathcal{H}_{t}$. A priori we do not assume that there is any relation between $\mathcal{H}_{t}$ and $\mathcal{F}_{t}$.

Definition 1.1 A portfolio process $\pi(t)$ is called $\mathcal{H}$-admissible if

$$
\begin{aligned}
& \pi(t) \quad \text { is } \mathcal{H}_{t} \text {-adapted } \\
& E\left[\int_{0}^{T}\left\{|\mu(t)-\rho(t)| \cdot|\pi(t)|+\sigma^{2}(t) \pi^{2}(t)+\int_{\mathbb{R}} \pi^{2}(t) \theta^{2}(t, z) \nu(d z)\right\} d t\right]<\infty \\
& \pi(t) \theta(t, z) \geq-1 \quad \text { a.s. for } d t \times \nu(d z)-\text { a.a. } t, z .
\end{aligned}
$$

If $\mathcal{H}_{t} \supset \mathcal{F}_{t}$ for some $t \in[0, T]$ we also add the condition

$\sigma(t) \pi(t)$ and $\theta(t, z) \pi(t)$ are caglad (i.e. left continuous with right limits)

and forward integrable with respect to $d B$ and $\tilde{N}(d t, d z)$, respectively

(see Section 4 for explanation and definition).

The set of all $\mathcal{H}$-admissible portfolios $\pi(\cdot)$ is denoted by $\mathcal{A}_{\mathcal{H}}$.

We now study the problem

Problem $1.2(\mathcal{H})$ Find $V_{\mathcal{H}}$ and $\pi^{*}=\pi_{\mathcal{H}}^{*} \in \mathcal{A}_{\mathcal{H}}$ such that

$$
V_{\mathcal{H}}=\sup _{\pi \in \mathcal{A}_{\mathcal{H}}} E\left[\ln X^{(\pi)}(T)\right]=E\left[\ln X^{\left(\pi^{*}\right)}(T)\right]
$$

The number $V_{\mathcal{H}}$ is called the value of the problem (1.18) and $\pi^{*}$ (if it exists) is called an optimal portfolio for (1.18).

We will discuss the following 3 cases separately:

(i) The classical case: $\mathcal{H}_{t}=\mathcal{F}_{t}$ for all $t$

(ii) The partial observation case: $\mathcal{H}_{t}=: \mathcal{E}_{t} \subseteq \mathcal{F}_{t}$ for all $t$

(iii) The insider case: $\mathcal{H}_{t}=: \mathcal{G}_{t} \supseteq \mathcal{F}_{t}$ for all $t$.

The presentation here is a survey based on recent joint works with Francesca Biagini (Biagini et al (2005)), Agnès Sulem (Øksendal et al (2004a)) and Giulia Di Nunno, Thilo Meyer-Brandis and Frank Proske (Di Nunno et al (2003), Di Nunno et al (2005)). 


\section{The classical case $\left(\mathcal{H}_{t}=\mathcal{F}_{t}\right)$}

In this case we get, by (1.12) and the Itô formula for semimartingales, that if $\pi \in \mathcal{A}_{\mathcal{F}}$ then the corresponding terminal wealth $X^{(\pi)}(T)$ is

$$
\begin{aligned}
X^{(\pi)}(T)= & \exp \left[\int _ { 0 } ^ { T } \left\{\rho(s)+(\mu(s)-\rho(s)) \pi(s)-\frac{1}{2} \sigma^{2}(s) \pi^{2}(s)\right.\right. \\
& \left.-\int_{\mathbb{R}}(\pi(s) \theta(s, z)-\ln (1+\pi(s) \theta(s, z))) \nu(d z)\right\} d s \\
& \left.+\int_{0}^{T} \pi(s) \sigma(s) d B(s)+\int_{0}^{T} \int_{\mathbb{R}} \ln (1+\pi(s) \theta(s, z)) \tilde{N}(d s, d z)\right]
\end{aligned}
$$

Hence, since the $d B(\cdot)$ - and the $\tilde{N}(\cdot, \cdot)$-integrals have expectation zero,

$$
\begin{aligned}
E\left[\ln X^{(\pi)}(T)\right]= & E\left[\int _ { 0 } ^ { T } \left\{\rho(s)+(\mu(s)-\rho(s)) \pi(s)-\frac{1}{2} \sigma^{2}(s) \pi^{2}(s)\right.\right. \\
& \left.\left.-\int_{\mathbb{R}}(\pi(s) \theta(s, z)-\ln (1+\pi(s) \theta(s, z))) \nu(d z)\right\} d s\right] .
\end{aligned}
$$

We can maximize this pointwise:

Fix $s, \omega$ and define the function $h: \mathbb{R} \rightarrow \mathbb{R}$ by

$$
h(\pi)=(\mu(s)-\rho(s)) \pi-\frac{1}{2} \sigma^{2}(s) \pi^{2}-\int_{\mathbb{R}}(\pi \theta(s, z)-\ln (1+\pi \theta(s, z))) \nu(d z) .
$$

This function is concave and hence we find its maximum by looking for a solution $\pi=\pi(s, \omega)$ of the equation

$$
0=h^{\prime}(\pi)=\mu(s)-\rho(s)-\sigma^{2}(s) \pi-\int_{\mathbb{R}}\left(\theta(s, z)-\frac{\theta(s, z)}{1+\pi \theta(s, z)}\right) \nu(d z)
$$

i.e.

$$
\sigma^{2}(s) \pi+\int_{\mathbb{R}} \frac{\pi \theta^{2}(s, z)}{1+\pi \theta(s, z)} \nu(d z)=\mu(s)-\rho(s)
$$

We have proved

Theorem 2.1 Suppose that for a.a. $(s, \omega)$ there exists a solution $\pi=\tilde{\pi}(s, \omega)$ of equation (2.3) and suppose that

$$
\tilde{\pi} \in \mathcal{A}_{\mathcal{F}}
$$

Then $\tilde{\pi}$ is optimal for Problem 1.2 $(\mathcal{F})$. 
In particular, in the continuous case $(\theta=0)$ we get

Corollary 2.2 Suppose $\theta=0$ and $\sigma(s) \neq 0$ a.s. for a.a. $s \in[0, T]$. Define

$$
\tilde{\pi}(s):=\frac{\mu(s)-\rho(s)}{\sigma^{2}(s)}
$$

a) If $\tilde{\pi} \in \mathcal{A}_{\mathcal{F}}$ then $\pi^{*}:=\pi_{\mathcal{F}}^{*}:=\tilde{\pi}$ is optimal for Problem 1.2 $(\mathcal{F})$.

b) If $\tilde{\pi} \in \mathcal{A}_{\mathcal{F}}$ then

$$
V_{\mathcal{F}}=E\left[\ln X^{(\tilde{\pi})}(T)\right]=E\left[\int_{0}^{T}\left\{\rho(s)+\frac{(\mu(s)-\rho(s))^{2}}{2 \sigma^{2}(s)}\right\} d s\right]
$$

\section{The partial observation case $\left(\mathcal{H}_{t} \subseteq \mathcal{F}_{t}\right)$}

In the partial observation case the trader at time $t$ does not have access to all the information $\mathcal{F}_{t}$ that can be obtained by observing the underlying Brownian motion $B(s) ; s \leq t$ and jump process $\tilde{N}([0, t], F) ; \bar{F} \in \mathbb{R} \backslash\{0\}$. This will be the situation if, for example, the trader only observes the stock prices $S(s) ; s \leq t$ and not the underlying processes. Indeed, this is a more realistic setup.

More generally, suppose the information available to the trader is represented by a filtration $\mathcal{H}_{t}$ such that

$$
\mathcal{E}_{t}:=\mathcal{H}_{t} \subset \mathcal{F}_{t} \quad \text { for all } t \in[0, T]
$$

What is the solution of Problem 1.2 then?

To answer this we proceed as follows:

Choose $\pi \in \mathcal{A}_{\mathcal{E}}$. Then by inserting an extra conditional expectation in (2.2) we get

$$
\begin{aligned}
& E\left[\ln x^{(\pi)}(T)\right]=E\left[\int _ { 0 } ^ { T } E \left[\rho(s)+(\mu(s)-\rho(s)) \pi(s)-\frac{1}{2} \sigma^{2}(s) \pi^{2}(s)\right.\right. \\
& \left.\left.\quad-\int_{\mathbb{R}}\{\pi(s) \theta(s, z)-\ln (1+\pi(s) \theta(s, z))\} \nu(d z) \mid \mathcal{E}_{s}\right] d s\right] \\
& =E\left[\int _ { 0 } ^ { T } \left\{\hat{\rho}(s)+(\hat{\mu}(s)-\hat{\rho}(s)) \pi(s)-\frac{1}{2} \widehat{\sigma^{2}(s)} \pi^{2}(s)\right.\right. \\
& \left.\left.\quad-\int_{\mathbb{R}}\left(\hat{\theta}(s, z) \pi(s)-E\left[\ln (1+y \theta(s, z)) \mid \mathcal{E}_{s}\right]_{y=\pi(s)}\right) \nu(d z)\right\} d s\right],
\end{aligned}
$$

where we have used the notation

$$
\hat{g}(s):=E\left[g(s) \mid \mathcal{E}_{s}\right] \quad \text { (the predictable version) }
$$

Again we can maximize this pointwise, for each $s \in[0, T]$ and $\omega \in \Omega$ : 
The concave function

$$
h(y):=(\hat{\mu}(s)-\hat{\rho}(s)) y-\frac{1}{2} \widehat{\sigma^{2}(s)} y^{2}-\int_{\mathbb{R}}\left(\hat{\theta}(s, z) y-E\left[\ln (1+y \theta(s, z)) \mid \mathcal{E}_{s}\right]\right) \nu(d z)
$$

is maximal when

$$
0=h^{\prime}(y)=\hat{\mu}(s)-\hat{\rho}(s)-\widehat{\sigma^{2}(s)} y-\int_{\mathbb{R}}\left(\hat{\theta}(s, z)-E\left[\frac{\theta(s, z)}{1+y \theta(s, z)} \mid \mathcal{E}_{s}\right]\right) \nu(d z)
$$

i.e. when $y=: \tilde{\pi}(s)$ solves the equation

$$
\widehat{\sigma^{2}}(s) y+\int_{\mathbb{R}} E\left[\frac{y \theta^{2}(s, z)}{1+y \theta(s, z)} \mid \mathcal{E}_{s}\right] \nu(d z)=\hat{\mu}(s)-\hat{\rho}(s) .
$$

This last deduction is based on differentiation within the conditional expectation, an operation which is justified if the following holds:

For a.a. $s, \omega$ the family of functions of $z$ defined by

$$
\left\{\frac{y \theta^{2}(s, z)}{1+y \theta(s, z)}\right\}_{y \theta(s, z) \geq-1}
$$

is uniformly integrable with respect to $\nu(d z)$.

We have proved:

Theorem 3.1 Assume that (3.6) holds and that for a.a. $s, \omega$ there exists a solution

$$
y=: \tilde{\pi}(s)
$$

of equation (3.5). Then

$$
\pi^{*}=\pi_{\mathcal{E}}^{*}:=\tilde{\pi}
$$

is optimal for Problem $1.2(\mathcal{E})$, provided that $\tilde{\pi} \in \mathcal{A}_{\mathcal{E}}$.

To get more explicit results we again specialize to the Brownian motion case:

Corollary 3.2 Suppose $\theta=0$. Define

$$
\tilde{\pi}(s)=\frac{\hat{\mu}(s)-\hat{\rho}(s)}{\widehat{\sigma^{2}}(s)} ; \quad s \in[0, T] .
$$

Suppose $\tilde{\pi} \in \mathcal{A}_{\mathcal{E}}$. Then $\pi^{*}:=\pi_{\mathcal{E}}^{*}:=\tilde{\pi}$ is optimal for Problem 1.2 $(\mathcal{E})$, and the corresponding value $V_{\mathcal{E}}$ is given by (see (3.3))

$$
\begin{aligned}
V_{\mathcal{E}} & =E\left[X^{(\tilde{\pi})}(T)\right] \\
& =E\left[\int_{0}^{T}\left\{\rho(s)+\frac{(\hat{\mu}(s)-\hat{\rho}(s))^{2}}{2 \widehat{\sigma^{2}}(s)}\right\} d s\right] .
\end{aligned}
$$

Combining (2.5) and (3.8) we get (if $\theta=0$ )

$$
V_{\mathcal{F}}-V_{\mathcal{E}}=\frac{1}{2} \int_{0}^{T} E\left[\frac{(\mu(s)-\rho(s))^{2}}{\sigma^{2}(s)}-\frac{(\hat{\mu}(s)-\hat{\rho}(s))^{2}}{\widehat{\sigma^{2}}(s)}\right] d s .
$$

This may be regarded as the value lost due to the reduced information $\mathcal{E}=\left\{\mathcal{E}_{t}\right\}_{t \in[0, T]}$ (compared to $\mathcal{F}=\left\{\mathcal{F}_{t}\right\}_{t \in[0, T]}$ ). 


\section{The insider case $\left(\mathcal{H}_{t}=: \mathcal{G}_{t} \supset \mathcal{F}_{t}\right)$}

We now consider the situation when the trader at time $t$ has access to more information than $\mathcal{F}_{t}$. For example, the trader may know the value of the stock at some future $T_{0}>T$. In this case the portfolio $\pi(t)$ is adapted to a larger filtration $\mathcal{H}_{t}=: \mathcal{G}_{t} \supset \mathcal{F}_{t}$ and the corresponding equation (1.12) for the wealth $X(t)=X^{(\pi)}(t)$ will be anticipating. Thus it will not necessarily make sense as an Itô stochastic differential equation, and we need to specify how to interpret the integral. To this end, suppose the trader applies a buy and hold strategy: She buys one stock at a time $\tau_{1} \geq 0$ (which may be random), keeps it until some (possibly random) time $\tau_{2} \in\left(\tau_{1}, T\right]$ when she sells it again. The money gained by this strategy (assuming that the risk free asset has the constant price 1 ) is

$$
S\left(\tau_{2}\right)-S\left(\tau_{1}\right)
$$

If we represent the portfolio by the number of stocks held at time $t, \varphi(t)$, then

$$
\varphi(t)=\mathcal{X}_{\left(\tau_{1}, \tau_{2}\right]}(t)= \begin{cases}1 & \text { if } \tau_{1}<t \leq \tau_{2} \\ 0 & \text { otherwise }\end{cases}
$$

and the money gained can be written as a limit of Riemann sums

$$
S\left(\tau_{2}\right)-S\left(\tau_{1}\right)=\lim _{\Delta t_{j} \rightarrow 0} \sum_{j=1}^{N} \varphi\left(t_{j}\right)\left(S\left(t_{j+1}\right)-S\left(t_{j}\right)\right)
$$

where $0=t_{0}<t_{1}<\cdots<t_{N}=T$ is a partition of $[0, T], \Delta t_{j}=t_{j+1}-t_{j}$ and $\varphi$ is evalueted at the left end point of each subinterval. Similarly, if the portfolio has the following form

$$
\varphi(t)=\sum_{i=0}^{M-1} a_{i} \mathcal{X}_{\left(\tau_{i}, \tau_{i+1}\right]}(t)
$$

where $a_{i} \in \mathbb{R}$ are constants and $0=\tau_{0} \leq \tau_{1} \leq \cdots \leq \tau_{M} \leq T$ are random buying/selling times, then we see that the money gained can be written

$$
\sum_{i=1}^{M-1} a_{i}\left(S\left(\tau_{i+1}\right)-S\left(\tau_{i}\right)\right)=\lim _{\Delta t_{j} \rightarrow 0} \sum_{j=1}^{N} \varphi\left(t_{j}\right) \Delta S\left(t_{j}\right),
$$

where $\Delta S\left(t_{j}\right)=S\left(t_{j+1}\right)-S\left(t_{j}\right)$.

This motivates that we use the forward integral in the modelling of the gains process in insider trading. This integral is defined as follows:

Definition 4.1 Let $\varphi(t)$ and $S(t)$ be two processes which are adapted to some filtration $\left\{\mathcal{H}_{t}\right\}_{0 \leq t \leq T}$. Assume that $S(t)$ is cadlag (right continuous with left limits) and that $\varphi(t)$ is caglad (left continuous with right limits). Then we define the forward integral of $\varphi$ with respect to $S$ by

$$
\int_{0}^{T} \varphi(t) d^{-} S(t):=\lim _{\Delta t_{j} \rightarrow 0} \sum_{j=1}^{N} \varphi\left(t_{j}\right) \Delta S\left(t_{j}\right),
$$

provided that the limit exists in probability and is independent of the partition chosen. 
We note that the forward integral with respect to a Lévy process $\eta(t)$ is an extension of the Itô integral, in the sense that if $\eta(\cdot)$ is a semimartingale with respect to $\left\{\mathcal{G}_{t}\right\}_{t \geq 0}$ then the two integrals coincide. We refer to Nualart et al (1988), Russo et al (1993, 2000), Biagini et al (2005) and Di Nunno et al (2005) for more information.

There is an Itô formula for forward integrals:

Theorem 4.2 (Russo et al (2000), Di Nunno et al (2005)) Let $Y(t):[0, T] \times \Omega \rightarrow \mathbb{R}$ be a stochastic process of the form

$$
Y(t)=y+\int_{0}^{t} \alpha(s) d s+\int_{0}^{t} \beta(s) d^{-} B(s)+\int_{0}^{t} \int_{\mathbb{R}} \gamma(s, z) \tilde{N}\left(d^{-} s, d z\right)
$$

or, in the usual shorthand notation,

$$
d^{-} Y(t)=\alpha(t) d t+\beta(t) d^{-} B(t)+\int_{\mathbb{R}} \gamma(t, z) \tilde{N}\left(d^{-} t, d z\right) ; \quad Y(0)=y
$$

Assume that $\gamma(t, z)$ is locally bounded in $z$ near $z=0$ for a.a. $t, \omega$ and that

$$
\int_{0}^{T} \int_{\mathbb{R}} \gamma(t, z)^{2} \nu(d z) d t<\infty \quad \text { a.s. }
$$

Let $f \in C^{1,2}(\mathbb{R} \times \mathbb{R})$ and define $Z(t)=f(t, Y(t))$. Then $Z(t)$ has the differential form

$$
\begin{aligned}
& d^{-} Z(t)=\frac{\partial f}{\partial t}(t, Y(t)) d t+\frac{\partial f}{\partial y}(t, Y(t)) d^{-} Y(t)+\frac{1}{2} \frac{\partial^{2} f}{\partial y^{2}}(t, Y(t)) \beta^{2}(t) d t \\
& \quad+\int_{\mathbb{R}}\left\{f\left(t, Y\left(t^{-}\right)+\gamma(t, z)\right)-f\left(t, Y\left(t^{-}\right)\right)-\frac{\partial f}{\partial y}\left(t, Y\left(t^{-}\right)\right) \gamma(t, z)\right\} \nu(d z) d t \\
& \quad+\int_{\mathbb{R}}\left\{f\left(t, Y\left(t^{-}\right)+\gamma(t, z)\right)-f\left(t, Y\left(t^{-}\right)\right)\right\} \tilde{N}\left(d^{-} t, d z\right) .
\end{aligned}
$$

In the Brownian motion case $(\gamma=0)$ this was proved by Russo et al (2000). Subsequently the extension to the general case above was proved by Di Nunno et al (2005). Note the similarity with the classical Itô formula in the adapted case (see e.g. Øksendal et al (2004b)).

In view of the above we use forward integrals in the equation for the wealth process $X(t)=X^{(\pi)}(t)$ corresponding to the portfolio $\pi$ of an insider, as follows (see (1.12)):

$$
\begin{aligned}
d X(t) & =X\left(t^{-}\right)\left[\{\rho(t)+(\mu(t)-\rho) \pi(t)\} d t+\sigma(t) \pi(t) d^{-} B(t)\right. \\
+ & \left.\pi(t) \int_{\mathbb{R}} \theta(t, z) \tilde{N}\left(d^{-} t, d z\right)\right] ; \quad 0 \leq t \leq T ; \quad X(0)=1 .
\end{aligned}
$$


By the Itô formula (Theorem 4.2) this equation has the solution (compare with (2.1))

$$
\begin{aligned}
X^{(\pi)}(t) & =\exp \left[\int _ { 0 } ^ { t } \left\{\rho(s)+(\mu(s)-\rho(s)) \pi(s)-\frac{1}{2} \sigma^{2}(s) \pi^{2}(s)\right.\right. \\
& \left.-\int_{\mathbb{R}}(\pi(s) \theta(s, z)-\ln (1+\pi(s) \theta(s, z))) \nu(d z)\right\} d s \\
& \left.+\int_{0}^{t} \pi(s) \sigma(s) d^{-} B(s)+\int_{0}^{t} \int_{\mathbb{R}} \ln (1+\pi(s) \theta(s, z)) \tilde{N}\left(d^{-} s, d z\right)\right] .
\end{aligned}
$$

Forward integrals do not in general have expectation 0 , so in this case we get a more complicated expression than (2.2):

$$
\begin{aligned}
& E\left[\ln X^{(\pi)}(T)\right]=E\left[\int _ { 0 } ^ { T } \left\{\rho(s)+(\mu(s)-\rho(s)) \pi(s)-\frac{1}{2} \sigma^{2}(s) \pi^{2}(s)\right.\right. \\
& \quad-\int_{\mathbb{R}}(\pi(s) \theta(s, z)-\ln (1+\pi(s) \theta(s, z))) \nu(z\} d s \\
& \left.+\int_{0}^{T} \pi(s) \sigma(s) d^{-} B(s)+\int_{0}^{T} \int_{\mathbb{R}} \ln (1+\pi(s) \theta(s, z)) \tilde{N}\left(d^{-} s, d z\right)\right] .
\end{aligned}
$$

Suppose there is a portfolio $\pi^{*} \in \mathcal{A}_{\mathcal{G}}$ which maximizes the function $J: \mathcal{A}_{\mathcal{G}} \rightarrow \mathbb{R}$ defined by

$$
J(\pi)=E\left[\ln X^{(\pi)}(T)\right]
$$

Moreover, suppose there exists $\epsilon>0$ such that

$$
\pi^{*}(t) \theta(t, z) \geq-1+\epsilon \quad \text { for } d t \times \nu(d z)-\text { a.a. } t, z \in[0, T] \times \mathbb{R} .
$$

Then for all bounded $\beta \in \mathcal{A}_{\mathcal{G}}$ there exists $\delta>0$ such that $\pi^{*}+y \beta \in \mathcal{A}_{\mathcal{G}}$ for all $|y| \leq \delta$ and the function

$$
g(y):=J\left(\pi^{*}+y \beta\right) ; \quad|y| \leq \delta
$$

is maximal for $y=0$. Therefore

$$
\begin{aligned}
0= & \left.\frac{d}{d y} g(y)\right|_{y=0}=E\left[\int _ { 0 } ^ { T } \left\{(\mu(s)-\rho(s)) \beta(s)-\sigma^{2}(s) \beta(s) \pi^{*}(s)\right.\right. \\
& \left.-\int_{\mathbb{R}}\left(\theta(s, z) \beta(s)-\frac{\theta(s, z) \beta(s)}{1+\pi^{*}(s) \theta(s, z)}\right) \nu(d z)\right\} d s \\
& \left.+\int_{0}^{T} \sigma(s) \beta(s) d^{-} B(s)+\int_{0}^{T} \int_{\mathbb{R}} \frac{\theta(s, z) \beta(s)}{1+\pi^{*}(s) \theta(s, z)} \tilde{N}\left(d^{-} s, d z\right)\right] .
\end{aligned}
$$


Now fix $t, h$ such that $t<t+h \leq T$ and apply this to

$$
\beta(s)=\gamma_{t}(\omega) \mathcal{X}_{[t, t+h]}(s) ; \quad s \in[0, T],
$$

where $\gamma_{t}(\omega)$ is a bounded $\mathcal{G}_{t}$-measurable random variable. Then we get

$$
\begin{gathered}
E\left[\left(\int_{t}^{t+h}\left\{\mu(s)-\rho(s)-\sigma^{2}(s) \pi^{*}(s)+\int_{\mathbb{R}} \frac{\pi^{*}(s) \theta^{2}(s, z)}{1+\pi^{*}(s) \theta(s, z)} \nu(d z)\right\} d s\right.\right. \\
\left.\left.\quad+\int_{t}^{t+h} \sigma(s) d B(s)+\int_{t}^{t+h} \frac{\theta(s, z)}{1+\pi^{*}(s) \theta(s, z)} \tilde{N}(d s, d z)\right) \gamma_{t}\right]=0
\end{gathered}
$$

Since this holds for all bounded $\mathcal{G}_{t}$-measurable $\gamma_{t}$ we deduce that

$$
\begin{gathered}
E\left[\left(\int_{t}^{t+h}\left\{\mu(s)-\rho(s)-\sigma^{2}(s) \pi^{*}(s)+\int_{\mathbb{R}} \frac{\pi^{*}(s) \theta^{2}(s, z)}{1+\pi^{*}(s) \theta(s, z)} \nu(d z)\right\} d s\right.\right. \\
\left.\left.\quad+\int_{t}^{t+h} \sigma(s) d B(s)+\int_{t}^{t+h} \frac{\theta(s, z)}{1+\pi^{*}(s) \theta(s, z)} \tilde{N}(d s, d z)\right) \mid \mathcal{G}_{t}\right]=0
\end{gathered}
$$

In other words, the process $M_{\pi}(t)$ defined by

$$
\begin{aligned}
M_{\pi}(t) & =\int_{0}^{t}\left\{\mu(s)-\rho(s)-\sigma^{2}(s) \pi(s)+\int_{\mathbb{R}} \frac{\pi(s) \theta^{2}(s, z)}{1+\pi(s) \theta(s, z)} \nu(d z)\right\} d s \\
& +\int_{0}^{t} \sigma(s) d B(s)+\int_{0}^{t} \frac{\theta(s, z)}{1+\pi(s) \theta(s, z)} \tilde{N}(d s, d z) ; \quad 0 \leq t \leq T
\end{aligned}
$$

is a martingale with respect to $\mathcal{G}=\left\{\mathcal{G}_{t}\right\}_{0 \leq t \leq T}$ if $\pi=\pi^{*}$. Thus we have proved the following special case of the result by Di Nunno et al (2003):

Theorem 4.3 (Di Nunno et al (2003)) Assume that there exists an optimal portfolio $\pi^{*}$ for Problem 1.2 $(\mathcal{G})$ and assume that $\pi^{*}$ satisfies (4.9). Then the process $M_{\pi^{*}}(t)$ defined by (4.11) is a martingale with respect to $\mathcal{G}$.

To get more explicit results, let us again consider the continuous case $(\theta=0)$ :

Corollary 4.4 (Biagini et al 2005)) Suppose $\theta=0$. Then a portfolio $\pi \in \mathcal{A}_{\mathcal{G}}$ is optimal for Problem 1.2 $(\mathcal{G})$ if and only if the process

$$
M_{\pi}^{(0)}(t):=\int_{0}^{t}\left\{\mu(s)-\rho(s)-\sigma^{2}(s) \pi(s)\right\} d s+\int_{0}^{t} \sigma(s) d B(s)
$$

is a martingale with respect to $\mathcal{G}$. 
Proof. We have already seen that if $\pi$ is optimal then $M_{\pi}^{(0)}(t)$ is a $\mathcal{G}$-martingale (Theorem 4.3). The converse follows from the fact that the map $F: \mathcal{A}_{\mathcal{G}} \rightarrow \mathbb{R}$ defined by

$$
F(\pi)=E\left[\ln X^{(\pi)}(T)\right]
$$

is concave. We refer to Biagini et al (2005) for details.

Corollary 4.5 Suppose that $\theta=0$ and $\sigma(t) \neq 0$ for a.a. $t \in[0, T]$. Then $\pi \in \mathcal{A}_{\mathcal{G}}$ is optimal for Problem 1.2 $(\mathcal{G})$ if and only if $B(t)$ is a semimartingale with respect to $\mathcal{G}$, and the semimartingale decomposition of $B$ with respect to $\mathcal{G}$ can be expressed in terms of $\pi$ by

$$
d B(t)=-\left\{\frac{\mu(t)-\rho(t)}{\sigma(t)}-\sigma(t) \pi(t)\right\} d t+d N_{\pi}(t)
$$

where

$$
d N_{\pi}(t)=\left\{\frac{\mu(t)-\rho(t)}{\sigma(t)}-\sigma(t) \pi(t)\right\} d t+d B(t)
$$

is a $\mathcal{G}$-martingale.

In specific examples the semimartingale decomposition of $B(\cdot)$ with respect to $\mathcal{G}$ is known, and this can then be used to find $\pi^{*}$.

A well-known case, first studied by Pikovski et al (1996) in the insider trading context, is the following:

\section{Example 4.6 Suppose}

$$
\mathcal{G}_{t}=\mathcal{F}_{t} \vee \sigma\left(B\left(T_{0}\right)\right) \quad \text { for some } T_{0}>T \text {. }
$$

This means that the insider knows the value of the underlying Brownian motion at some future time $T_{0}$. (If the coefficients $\mu$ and $\sigma$ are known constants, then this is the same as knowing the value $S\left(T_{0}\right)$ of the stock at time $T_{0}$.) In this case it is known, by a result of Itô $[\mathrm{I}]$, that $B(\cdot)$ is a semimartingale with respect to $\mathcal{G}$ and its semimartingale decomposition is

$$
d B(t)=\frac{B\left(T_{0}\right)-B(t)}{T_{0}-t} d t+d \hat{B}(t)
$$

where $\hat{B}(t)$ is a $\mathcal{G}$-martingale (in fact, a $\mathcal{G}$-Brownian motion). Comparing (4.16) and (4.13) we conclude, by uniqueness of the semimartingale decomposition, that

$$
\sigma(t) \pi^{*}(t)-\frac{\mu(t)-\rho(t)}{\sigma(t)}=\frac{B\left(T_{0}\right)-B(t)}{T_{0}-t}
$$

i.e.

$$
\pi^{*}(t)=\pi_{\mathcal{G}}^{*}(t)=\frac{\mu(t)-\rho(t)}{\sigma^{2}(t)}+\frac{B\left(T_{0}\right)-B(t)}{\sigma(t)\left(T_{0}-t\right)}
$$

Thus we have 
Corollary 4.7 Suppose $\theta=0, \sigma(t) \neq 0$ for a.a. $t \in[0, T]$ and that

$$
\mathcal{G}_{t}=\mathcal{F}_{t} \vee \sigma\left(B\left(T_{0}\right)\right) \quad \text { for some } T_{0}>T \text {. }
$$

Then the optimal portfolio $\pi^{*} \in \mathcal{A}_{\mathcal{G}}$ for Problem 1.2 (G) is given by (4.17). The corresponding value $V_{\mathcal{G}}$ is

$$
V_{\mathcal{G}}=V_{\mathcal{F}}+\frac{1}{2} \ln \frac{T_{0}}{T_{0}-T},
$$

and hence the value gained by the information about $B\left(T_{0}\right)$ is

$$
V_{\mathcal{G}}-V_{\mathcal{F}}=\frac{1}{2} \ln \frac{T_{0}}{T_{0}-T} .
$$

\section{Remark 4.8}

(i) Note that the optimal insider portfolio $\pi_{\mathcal{G}}^{*}$ in (4.17) differs from the optimal portfolio $\pi_{\mathcal{F}}^{*}$ in $(2.4)$ of the honest trader by the term

$$
\pi_{\mathcal{G}}^{*}(t)-\pi_{\mathcal{F}}^{*}(t)=\frac{B\left(T_{0}\right)-B(t)}{\sigma(t)\left(T_{0}-t\right)} ; \quad 0 \leq t \leq T .
$$

This term is not available to the honest trader, because it is not $\mathcal{F}_{t^{-}}$adapted.

(ii) Note that $V_{\mathcal{G}} \rightarrow \infty$ as $T_{0} \rightarrow T$. However, if $T_{0}=T$ it is impossible for the insider to trade optimally, because of the term in (4.20), which behaves like the (non-existing!) derivative at $B(t)$ at $t=T_{0}$ as $t \rightarrow T_{0}^{-}$.

\section{References}

Applebaum, D. 2004: Lévy Processes and Stochastic Calculus. Cambridge University Press.

Barndorff-Nielsen, O. 1998: Processes of normal inverse Gaussian type. Finance and Stochastics 1, 41-68.

Biagini, F. and Øksendal, B. 2005: A general stochastic calculus approach to insider trading. Applied Mathematics and Optimization (to appear).

Di Nunno, G., Meyer-Brandis, T., Øksendal, B. and Proske, F. 2005: Malliavin calculus and anticipative Itô formulae for Lévy processes. Infinite Dimensional Analysis, Quantum Probability and Related Topics (to appear).

Di Nunno, G., Meyer-Brandis, T., Øksendal, B. and Proske, F. 2003: Optimal portfolio for an insider in a market driven by Lévy processes. Preprint, Dept. of Math., Univ. of Oslo $36 / 2003$.

Eberlein, E. 2001: Application of generalized hyperbolic Lévy motion to finance. In Barndorff-Nielsen, O. 2001 (ed.): Lévy Processes. Birkhäuser, pp. 319-336. 
Itô, K. 1978: Extension of stochastic integrals. In Proceedings of International Symposium on Stochastic Differential Equations. Wiley, pp. 95-109.

Jacod, J. and Shiryaev, A. 2003: Limit Theorems for Stochastic Processes. Second Edition. Springer.

Nualart, D. and Pardoux, E. 1988: Stochastic calculus with anticipating integrands. Probability Theory and Related Fields 78, 535-581.

Øksendal, B. and Sulem, A. 2004a: Partial observation control in an anticipating environment. Russian Math. Surveys 59, 355-375.

Øksendal, B. and Sulem, A. 2004b: Applied Stochastic Control of Jump Diffusions. Springer.

Pikovsky, I. and Karatzas, I. 1996: Anticipating portfolio optimization. Advances in Applied Probability 28, 1095-1122.

Russo, F. and Vallois, P. 1993: Forward, backward and symmetric stochastic integration. Probability Theory and Related Fields 97, 403-421.

Russo, F. and Vallois, P. 2000: Stochastic calculus with respect to continuous finite variation processes. Stochastics and Stochastics Reports 70, 1-40.

Schoutens, W. 2003: Lévy Processes in Finance. Wiley. 